\title{
Ações internas voltadas ao fomento da inovação: as empresas também devem fazer sua "lição de casa"
}

\author{
Tales Andreassi*
}

\begin{abstract}
Resumo
É fato indiscutível a importância da participação governamental no estabelecimento de um sistema nacional de inovação eficiente e capaz de gerar uma infraestrutura básica que faça aflorar a P\&D empresarial. Muito se cobra do Governo brasileiro 0 estabelecimento de programas e políticas de apoio à inovação tecnológica e, sem dúvida, essa é uma condição fundamental para atingirmos um patamar mais elevado no que diz respeito ao nosso desenvolvimento. Contudo, esperar que só o Governo faça sua parte é ingênuo e seguramente não trará os resultados esperados. As empresas precisam desenvolver uma série de políticas, programas e ações que efetivamente contribuam para um melhor resultado inovativo. Ou seja, precisam fazer sua "lição de casa". 0 objetivo desse artigo é discutir algumas ações que as empresas devem tomar para ter um melhor resultado proveniente do seu esforço inovativo.
\end{abstract}

\section{Palavras-chave: inovação, pesquisa e desenvolvimento, políticas de P\&D}

\begin{abstract}
Government has a fundamental role in the development of an efficient national innovation system, strong enough to allow the development of $R \& D$ and innovation in firms. Thus companies constantly expect several programs and policies from Government in order to support their technological innovation. This is a fundamental condition if Brazil wants to reach a higher level of development. However, not only the Government, but also the companies need to develop some actions for a better innovative result, i.e., companies need to do their "homework". The objective of this paper is to discuss and analyze some of these actions to support the innovation in companies.
\end{abstract}

Key- words: innovation, R\&D, R\&D policies

\section{Introdução}

É indiscutível a importância da participação governamental no estabelecimento de um Sistema Nacional de Inovação (SNI) eficaz e capaz de gerar uma infra-estrutura básica que propicie a pesquisa e desenvolvimento (P\&D) empresarial. Uma das explicações para o estágio em que se encontra a inovação no Brasil é que o nosso SNI é pouco articulado. Por sua vez, o conceito de SNI foi desenvolvido simultaneamente por Chris Freeman, na Inglaterra, e Richard Nelson, nos EUA, em 1988, e pode ser entendido como "uma construção institucional, produto de uma ação planejada e consciente ou de um somatório de decisões não planejadas e desarticuladas, que impulsiona o progresso tecnológico em economias capitalistas complexas" (ALBUQUERQUE, 1995). Nesse sentido, o exemplo da Coréia do Sul é um dos mais citados, com suas políticas de incentivo fiscal, estímulo ao emprego de pesquisadores estrangeiros e cooperação universidade/empresa, entre outras iniciativas.

O governo brasileiro é muito cobrado para que estabeleça programas e políticas de apoio à inovação tecnológica, e sem dúvida isso é condição fundamental para atingirmos um patamar mais elevado de desenvolvimento econômico e social. Contudo, acreditar que só o governo deve fazer sua parte, seguramente, não trará os resultados esperados. As empresas precisam desenvolver, internamente, uma série de políticas, programas e ações que contribuam para um melhor resultado inovador; ou seja, precisam fazer sua "lição de casa".

\footnotetext{
* Professor da Escola de Administração de Empresas de São Paulo (EAESP), Fundação Getulio Vargas (FGV). E-mail: tandreassi@ fgvsp.br. Endereço: Av. 9 de Julho, 2029 - Bela Vista. CEP: 01313-902 - São Paulo- SP

Artigo aceito para publicação em novembro de 2004 e aceito em março de 2005.
} 
O objetivo deste artigo é discutir algumas ações a serem empreendidas pelas empresas para que consigam um melhor resultado nos seus esforços pela inovação. É um artigo eminentemente teórico, recorrendo a fontes secundárias conseguidas durante pesquisas empíricas em empresas que investem em inovação tecnológica.

\section{Metodologia}

Apesar do cunho eminentemente teórico deste artigo - uma vez que só foram utilizadas fontes secundárias -, todo o raciocínio lógico foi baseado em estudos que tiveram como ponto de partida os dados coletados anualmente pela Associação Nacional de Pesquisa, Desenvolvimento e Engenharia das Empresas Inovadoras (Anpei), desde 1992, e que compõem sua Base de Dados sobre Indicadores Empresarias de Inovação Tecnológica. Trata-se de um projeto de longo prazo da associação (com apoio do Sebrae, Finep e Fapesp), cujo objetivo é mensurar indicadores da área de pesquisa e desenvolvimento empresarial e servir como referência em futuros estudos, seja como guia para as empresas tomarem decisões na área de $\mathrm{P} \& \mathrm{D}$ ou como subsídio para políticas específicas nessa área. Hoje, mais de 1.300 empresas já participaram da base de dados da Anpei, na condição de informantes. É certo que essa base de dados apresenta vieses e problemas de ordem metodológica, mas não se pode negar que sua implantação foi criteriosa, no sentido de minimizar o aparecimento de tais vieses (ANPEI, 1997).

\section{Comprar ou gerar tecnologia?}

Uma questão que aflige as empresas é ter de optar entre a compra (difusão) e o investimento em esforço interno de pesquisa e desenvolvimento. A relação entre comprar e produzir tecnologia é abordada de duas formas distintas pela literatura afim. Uma primeira abordagem, defendida pelos economistas clássicos, sugere que essa relação deve ser substitutiva, ou seja, a empresa deve decidir entre comprar ou produzir a tecnologia de que necessita. Contudo, tal abordagem não é passível de críticas, conforme atestam Patel e Pavitt (1995) dado que nenhum país detém a maioria da tecnologia disponível no mundo, seria errado assumir que P\&D endógena e compra de tecnologia estrangeira sejam substitutos perfeitos para se obter a melhor tecnologia disponível em determinado momento.

Patel \& Pavitt (1995) também se referem a alguns dados coletados pela Organização para Cooperação e Desenvolvimento Econômico (OCDE) que mostram a relação entre os valores destinados à compra e aqueles direcionados para o financiamento da pesquisa e desenvolvimento, conforme consta da Tabela 1. A tabela mostra que, mesmo países desenvolvidos com $\mathrm{P} \& \mathrm{D}$ de alta qualidade compram tecnologia de outros países.

Tabela 1

Relação percentual entre atividades relacionadas com compra de tecnologia e gasto total em pesquisa e desenvolvimento

\begin{tabular}{lcccccccc}
\hline Países & $\mathbf{1 9 7 5}$ & $\mathbf{1 9 7 7}$ & $\mathbf{1 9 7 9}$ & $\mathbf{1 9 8 1}$ & $\mathbf{1 9 8 3}$ & $\mathbf{1 9 8 5}$ & $\mathbf{1 9 8 7}$ & $\mathbf{1 9 8 9}$ \\
\hline Canadá & 0,38 & 0,41 & 0,33 & 0,29 & 0,32 & 0,28 & 0,26 & 0,27 \\
Dinamarca & 0,45 & 0,54 & 0,63 & 0,27 & 0,45 & 0,46 & - & - \\
Finlândia & 0,44 & 0,39 & 0,36 & 0,28 & 0,24 & 0,22 & 0,22 & 0,21 \\
França & 0,29 & 0,29 & 0,31 & 0,21 & 0,20 & 0,22 & 0,21 & 0,20 \\
Alemanha & 0,24 & 0,22 & 0,21 & 0,16 & 0,16 & 0,16 & 0,21 & 0,25 \\
Itália & 0,37 & 0,38 & 0,34 & 0,33 & 0,34 & 0,26 & 0,21 & 0,21 \\
Japão & 0,10 & 0,10 & 0,12 & 0,07 & 0,06 & 0,05 & 0,04 & 0,04 \\
Noruega & 0,44 & 0,38 & 0,43 & 0,27 & 0,26 & 0,16 & 0,27 & 0,18 \\
Reino Unido & 0,22 & - & - & 0,17 & 0,18 & 0,21 & 0,24 & 0,24 \\
\multicolumn{1}{r}{ EUA } & 0,03 & 0,03 & 0,03 & 0,02 & 0,02 & 0,02 & 0,03 & 0,04 \\
\hline
\end{tabular}

Fonte: Patel \& Pavitt (1995), com base em dados da OCDE. 
A outra abordagem sugere que existe uma relação complementar entre difusão e inovação. A idéia de que os países em desenvolvimento devam se concentrar na exploração da tecnologia já desenvolvida pelos países industrializados é errônea. Bell \& Pavitt (1995) sugerem que gerar mudança tecnológica requer um considerável esforço doméstico tanto quanto o necessário à aquisição de tecnologia importada. Nesse sentido, Cohen \& Levinthal (1989) também argumentam que pesquisa e desenvolvimento não somente gera inovação como também desenvolve a habilidade da empresa identificar, assimilar e explorar o conhecimento do ambiente. Freeman \& Soete (1997) notam que para assimilar qualquer tecnologia sofisticada e operá-la com eficiência é necessário ter uma capacitação em $\mathrm{P} \& \mathrm{D}$, ainda que adaptativa.

Possuir uma equipe interna de pesquisa e desenvolvimento não somente é importante para uma melhor adaptação da tecnologia comprada, como o oposto também é verdadeiro. Ou seja, a compra da tecnologia também auxilia o esforço interno de $\mathrm{P} \& \mathrm{D}$, pois caso contrário a empresa pode estar incorrendo no risco de "reinventar a roda". Empresas no leste asiático utilizam a transferência internacional de tecnologia para capacitação do seu quadro de pessoal, em função do acesso que passam a ter a informações de design e rotinas de produção.

Um estudo específico sobre esse tema foi conduzido por Odagiri (1983), que analisou a relação entre gastos em P\&D e pagamento de royalties em 370 empresas japonesas. $\mathrm{O}$ autor verificou uma correlação positiva entre essas duas variáveis, concluindo que empresas com um alto esforço de pesquisa e desenvolvimento tendem a pagar mais royalties pela aquisição de tecnologia.

Bell \& Pavitt (1993) encontraram resultados interessantes ao comparar alguns países em desenvolvimento. Nos países de industrialização recente do leste asiático, a importação de tecnologia é complementada com um enorme esforço para desenvolver capacitações locais, como por exemplo:

- acordos de transferência de tecnologia com companhias estrangeiras que cobrem não somente a aquisição de competências em operação e manutenção, mas também em design, engenharia e administração de projetos;

- altos investimentos em educação em nível de pós-graduação e em programas de treinamento em países desenvolvidos, voltados para engenheiros e gerentes, os quais acabam acessando importantes networks internacionais;

- no caso de algumas empresas, o estabelecimento de centros de pesquisa e desenvolvimento ou aquisição de firmas no exterior, para ter acesso a habilidades específicas.

No Brasil, contudo, não é verificada essa relação complementar entre compra e esforço interno de P\&D. Andreassi (1998), baseado em dados de 272 empresas que constavam da base de dados da Anpei em 1994, estabeleceu correlações entre as variáveis "dispêndios em P\&D por vendas" e "dispêndios em aquisição de tecnologia por vendas", chegando aos seguintes resultados:

Tabela 2

Matriz de correlação para as 272 empresas consideradas

\begin{tabular}{lcc}
$\begin{array}{c}\text { GASTOS COM } \\
\text { INOVAÇÃO }(\mathbf{N}=\mathbf{2 7 2})\end{array}$ & $\begin{array}{c}\text { P\&D POR VENDAS } \\
(\%)\end{array}$ & $\begin{array}{c}\text { AQUISIÇÃO DE } \\
\text { TECNOLOGIA POR } \\
\text { VENDAS (\%) }\end{array}$ \\
\hline P\&D por vendas $(\%)$ & $\mathrm{r}=1,00$ & $\begin{array}{c}\mathrm{r}=0,05 \\
\mathrm{p}=0,372\end{array}$ \\
\hline $\begin{array}{l}\text { Aquisição de tecnologia por } \\
\text { vendas (\%) }\end{array}$ & $\mathrm{r}=0,05$ & $\mathrm{r}=1,00$ \\
\hline
\end{tabular}

( $\mathrm{r}$ = coeficiente da correlação de Spearman; $\mathrm{N}$ = número de empresas; $\mathrm{p}$ = nível de significância)

Fonte: Andreassi (1998)

A Tabela 2 mostra que o dispêndio em aquisição de tecnologia por vendas apresenta uma correlação fraca e não significativa com o dispêndio em P\&D por vendas. Para uma análise mais aprofundada, foi adotado o mesmo procedimento, estratificando-se a amostra por setor e porte das empresas, conforme as Tabelas 3 e 4. 
Tabela 3

Matriz de correlação por setor

\begin{tabular}{|c|c|}
\hline SETOR & $\begin{array}{l}\text { Correlação entre } \\
\text { P\&D e aquisição de tecnologia }\end{array}$ \\
\hline Agricultura e extrativismo $(n=12)$ & $r=0,11 ; p=0,744$ \\
\hline Alimentos $(n=9)$ & $r=-0,26 ; p=0,493$ \\
\hline Cerâmica, vidro e produtos correlatos $(n=14)$ & $\mathrm{r}=-0,05 ; \mathrm{p}=0,869$ \\
\hline Equipamentos eletroeletrônicos $(n=34)$ & $r=0,03 ; p=0,880$ \\
\hline Instrumentos ópticos, médicos e de precisão $(\mathrm{n}=9)$ & $\mathrm{r}=0,83 ; \mathrm{p}=0,005$ \\
\hline Máquinas industriais e equipamentos $(\mathrm{n}=46)$ & $r=-0,08 ; p=0,604$ \\
\hline Metais primários $(\mathrm{n}=22)$ & $r=-0,10 ; p=0,674$ \\
\hline Papel e celulose $(\mathrm{n}=11)$ & $\mathrm{r}=0,27 ; \mathrm{p}=0,414$ \\
\hline Polímeros $(\mathrm{n}=12)$ & $\mathrm{r}=-0,14 ; \mathrm{p}=0,657$ \\
\hline Produtos de metais $(\mathrm{n}=21)$ & $r=0,03 ; p=0,887$ \\
\hline Produtos de transportes $(\mathrm{n}=19)$ & $\mathrm{r}=0,25 ; \mathrm{p}=0,298$ \\
\hline Químicos $(\mathrm{n}=52)$ & $r=0,23 ; p=0,105$ \\
\hline Outros $(n=11)$ & $r=-0,02 ; p=0,995$ \\
\hline \multicolumn{2}{|c|}{$(\mathrm{r}=$ coeficiente da correlação de Spearman; $\mathrm{n}$ = número de empresas; $\mathrm{p}$ = nível de significância) } \\
\hline \multicolumn{2}{|l|}{ Fonte: Andreassi (1998). } \\
\hline \multicolumn{2}{|c|}{$\begin{array}{c}\text { Tabela } 4 \\
\text { Matriz de correlação por tamanho da empresa }\end{array}$} \\
\hline TAMANHO & $\begin{array}{c}\text { Correlação entre } \\
\text { P\&D e aquisição de tecnologia }\end{array}$ \\
\hline $\begin{array}{l}\text { Micro e pequenas empresas } \\
\text { (até } 99 \text { funcionários, } n=64 \text { ) }\end{array}$ & $r=0,02 ; p=0,890$ \\
\hline $\begin{array}{l}\text { Médias empresas } \\
(100 \text { a } 499 \text { funcionários, } \mathrm{n}=96)\end{array}$ & $r=0,05 ; p=0,625$ \\
\hline $\begin{array}{l}\text { Grandes e macro empresas } \\
\text { (mais de } 500 \text { funcionários, } n=112 \text { ) }\end{array}$ & $r=0,12 ; p=0,228$ \\
\hline
\end{tabular}

$(\mathrm{r}=$ coeficiente da correlação de Spearman; $\mathrm{n}=$ número de empresas; $\mathrm{p}=$ nível de significância $)$

Fonte: Andreassi (1998).

Pela Tabela 3, pode-se notar que em apenas um setor, o de instrumentos ópticos, médicos e de precisão, a correlação entre compra e esforço de P\&D foi estatisticamente significativa. Quanto ao tamanho da empresa, a Tabela 4 mostra que não foi encontrada correlação significativa em nenhum dos estratos considerados.

Os resultados encontrados evidenciam que as empresas brasileiras estão se concentrando apenas nas atividades de compra de tecnologia ou nas atividades de desenvolvimento de esforço inovador interno. Isso pode sugerir uma disfunção no sistema de inovação brasileiro, uma vez que a literatura preconiza uma relação complementar entre essas duas variáveis.

\section{P\&D e faturamento da empresa}

Outro ponto fundamental nas estratégias de uma empresa é o quanto investir em inovação. Certamente, esse valor irá depender do setor de atividade, das estratégias corporativas e do quanto a alta administração está sensibilizada para essa questão, entre outros fatores. Entretanto, o que importa frisar é que os resultados derivados de investimentos em $\mathrm{P} \& \mathrm{D}$ aparecem no longo prazo e, portanto, a empresa deve manter uma política de investi- 
mentos em pesquisa e desenvolvimento consolidada e razoavelmente constante ao longo do tempo. Condicionar os investimentos em $\mathrm{P} \& \mathrm{D}$ aos resultados obtidos pela empresa é algo que pode prejudicá-la a longo prazo.

Odagiri (1983), analisando 370 empresas japonesas, estabeleceu correlações entre a taxa de crescimento no faturamento no período 1969-81 e os gastos em P\&D por faturamento nos períodos 1969-72 e 1978-81. Os resultados mostram que, em geral, é fraca a correlação entre o crescimento no faturamento e os gastos em P\&D por faturamento, tanto em períodos anteriores quanto posteriores. Porém chama a atenção que, considerando a amostra total e os setores mais inovadores, a correlação entre o crescimento no faturamento e os gastos em $\mathrm{P} \& \mathrm{D}$ por faturamento em períodos precedentes é pouco superior à correlação entre o crescimento no faturamento e os gastos em $\mathrm{P} \& \mathrm{D}$ por faturamento em períodos posteriores, indicando que aparentemente é a intensidade em $P \& D$ que leva a um crescimento no faturamento e não o oposto. Outros estudos que chegaram a resultados semelhantes foram desenvolvidos por Morbey \& Reithner (1990), Dugal \& Morbey (1995) e Brenner \& Rushton (1989) e Franko (1989).

Morbey \& Reithner (1990, p.12) concluíram que "a P\&D tem um papel importante no crescimento das empresas, embora se reconheça que muitos outros fatores também afetam tal crescimento". Já Dugal \& Morbey (1995), estudando períodos recessivos, verificaram que o declínio no faturamento em períodos recessivos é menos típico em empresas que investiram significativamente em pesquisa e desenvolvimento, chegando a estipular que esse valor de gastos em P\&D por faturamento estaria acima de $3 \%$.

Brenner \& Rushton (1989) estudaram especificamente o setor químico, concluindo que a maioria das empresas com crescimento no faturamento acima da média também apresentaram gastos em P\&D por faturamento em períodos precedentes acima da média. Da mesma forma, empresas com um crescimento no faturamento abaixo da média também apresentaram gastos em pesquisa e desenvolvimento por faturamento em períodos precedentes abaixo da média. Por fim, Franko (1989) examinou as 83 maiores empresas mundiais, encontrando uma correlação positiva entre P\&D por faturamento e o crescimento do faturamento. Um estudo similar foi conduzido em 125 empresas brasileiras por Andreassi (1998), conforme pode ser examinado nas Tabelas 5 e 6 :

Tabela 5

\begin{tabular}{|c|c|}
\hline $\begin{array}{l}\text { Indicadores } \\
\text { Estratos }\end{array}$ & $\begin{array}{c}\text { P\&D/faturamento (1994) e } \\
\text { Evolução do faturamento (1994-96) }\end{array}$ \\
\hline & $\mathbf{r} /(\mathbf{n}) / \mathbf{p}$ \\
\hline Todas empresas & $0,262(125)<1 \%$ \\
\hline Químico & $-0,023(22) \mathrm{ns}$ \\
\hline Máquinas industriais & $0,358(18) \mathrm{ns}$ \\
\hline Eletroeletrônico & $0,057(18) \mathrm{ns}$ \\
\hline Metal primário & 0,467 (10) ns \\
\hline Porte pequeno & $0,133(18) \mathrm{ns}$ \\
\hline Porte médio & $0,204(40) \mathrm{ns}$ \\
\hline Porte grande & $0,153(67) \mathrm{ns}$ \\
\hline Capital nacional & $0,268(105)<1 \%$ \\
\hline Capital estrangeiro & $0,263(20) \mathrm{ns}$ \\
\hline
\end{tabular}

$(\mathrm{r}=$ coeficiente da correlação de Spearman; $\mathrm{n}$ = número de empresas; $\mathrm{p}=$ nível de significância; $\mathrm{ns}$ = não-significativo $)$

Fonte: Andreassi (1999). 
Tabela 6

Correlação entre o faturamento e a despesa em $P \& D$ em períodos subseqüentes

\begin{tabular}{lc}
\hline $\begin{array}{l}\text { Indicadores } \\
\text { Estratos }\end{array}$ & P\&D/faturamento (1996) e \\
Evolução do faturamento (1994-96)
\end{tabular}

Estratos

$\mathbf{r} /(\mathbf{n}) / \mathbf{p}$

\begin{tabular}{lc}
\hline Todas empresas & $0,365(125)<1 \%$ \\
\hline Químico & $0,386(22)<10 \%$ \\
\hline Máquinas industriais & $0,251(18) \mathrm{ns}$ \\
\hline Eletroeletrônico & $0,232(18) \mathrm{ns}$ \\
\hline Metal primário & $0,442(10) \mathrm{ns}$ \\
\hline Porte pequeno & $0,259(18) \mathrm{ns}$ \\
\hline Porte médio & $0,288(40)<10 \%$ \\
\hline Porte grande & $0,291(67)<5 \%$ \\
\hline Capital nacional & $0,343(105)<1 \%$ \\
\hline Capital estrangeiro & $0,492(20)<5 \%$
\end{tabular}

$(\mathrm{r}=$ coeficiente da correlação de Spearman; $\mathrm{n}$ = número de empresas; $\mathrm{p}$ = nível de significância; $\mathrm{ns}$ = não-significativo $)$

Fonte: Andreassi (1999).

A Tabela 5 aborda a relação entre despesa em pesquisa e desenvolvimento em períodos precedentes e a evolução no faturamento bruto da empresa, considerando a despesa em P\&D por faturamento relativa ao anobase 1994 e a evolução do faturamento no ano-base 1996 em relação a 1994. Verifica-se significativa correlação positiva apenas na amostra total e no estrato capital nacional. A análise das demais estratificações revela, com exceção do setor químico, que os coeficientes de correlação são positivos, mas não o suficiente para terem maior expressão em termos estatísticos.

Já a Tabela 6 mostra a relação entre faturamento e despesa em pesquisa e desenvolvimento em períodos subseqüentes, considerando a despesa em P\&D por faturamento referente ao ano-base 1996 e a evolução do faturamento no período 1994-96. Verifica-se uma significativa correlação positiva entre as variáveis na amostra total, bem como nos estratos "setor químico", "porte médio" e "grande", "capital nacional" e "estrangeiro". Comparando a Tabela 6 com a Tabela 5, percebe-se que, além de um maior número de estratos com correlação positiva significativa, os coeficientes de correlação são maiores na Tabela 6. Assim, pode-se inferir que, de um modo geral, a correlação entre a evolução no faturamento e a despesa em P\&D por faturamento em períodos subseqüentes é significativamente mais forte que a correlação entre a despesa em P\&D em períodos precedentes e a evolução no faturamento.

Os resultados encontrados na literatura mostram justamente o contrário, conforme mencionado anteriormente. Exemplificando, Odagiri (1983) encontrou uma correlação mais forte entre gastos em pesquisa e desenvolvimento em períodos precedentes e evolução no faturamento $(r=0,222, p<5 \%)$ do que entre evolução no faturamento e gastos em $P \& D$ em períodos subseqüentes $(r=0,173, p<10 \%)$. Tal resultado sinaliza que, no Brasil, é a despesa em P\&D que está atrelada ao faturamento. Isso significa que os investimentos em pesquisa e desenvolvimento ainda estão muito condicionados aos bons resultados alcançados pela empresa, o que não é recomendável. Pelo fato da P\&D ser estratégica para a empresa, com resultados eminentemente de longo prazo, o ideal seria que, na medida do possível, o orçamento a ela destinado não sofresse grandes alterações de um ano para outro, para que as linhas de trabalho previamente traçadas não sofressem alterações ou interrupções. 


\section{Equipe interna em P\&D}

Finalmente, o terceiro ponto a ser abordado é sobre a importância da empresa manter uma equipe técnica treinada e especializada em pesquisa e desenvolvimento. Para tanto, a análise de Andreassi e Sbragia (2001) examinou os fatores determinantes do grau de "inovatividade" das empresas brasileiras mais e menos inovadoras, assim classificadas segundo o percentual do faturamento conseguido com produtos novos ou aperfeiçoados.

O autor utilizou uma amostra de 141 empresas industriais, de diferentes setores, e aplicou a técnica da análise discriminante multivariada para obter os fatores que discriminam o grau de "inovatividade" dessas empresas.

No final da década de 1980, acreditava-se que o número de produtos lançados no mercado cresceria assustadoramente nas décadas seguintes, conforme relata Cooper e Kleinschmidt (1996). No entanto, o que se viu foi que muito embora o nível de lançamento de novos produtos tenha realmente crescido, o número de fracassos também aumentou a taxas alarmantes. Previsões indicam que mais da metade dos recursos alocados em inovações é desperdiçada e que cerca de $75 \%$ de todas as criações e $33 \%$ de todos os lançamentos fracassam comercialmente.

Mas, afinal, que características essas empresas que conseguem lançar produtos com sucesso, aqui chamadas de "empresas mais inovadoras", possuem em relação àquelas que não conseguem a mesma taxa de sucesso em seus lançamentos, denominadas nesse estudo de "empresas menos inovadoras"?

As variáveis independentes métricas testadas na análise discriminante são:

- despesas em P\&D por faturamento;

- número de doutores, mestres e graduados por número de funcionários;

- número de doutores por número de funcionários;

- número de patentes depositadas e/ou concedidas por número de funcionários;

- percentual de projetos de inovação finalizados;

- faturamento por número de funcionários;

- lucro líquido por número de funcionários;

- redução de custos decorrentes de melhorias de processo por número de funcionários;

- participação no mercado nacional.

A técnica de análise discriminante mais adequada nesse caso é a stepwise, que começa com a exclusão de todas as variáveis independentes do modelo (ou seja, até agora nenhuma variável independente discrimina a dependente) e a seleção da variável que maximiza a distância entre os grupos. A Tabela 7 apresenta as distâncias quadráticas e a estatística $F$ para cada variável. 
Tabela 7

Distância quadrática e estatística $\mathrm{F}$ para o step 0

\begin{tabular}{lcc}
\hline \multicolumn{1}{c}{ Variável } & $\begin{array}{c}\text { Distância } \\
\text { quadrática }\end{array}$ & Estatística $\boldsymbol{F}$ \\
\hline Despesas em P\&D por faturamento (\%) & 0,277 & 4,558 \\
\hline $\begin{array}{l}\text { Número de doutores, mestres e graduados por número } \\
\text { de funcionários (\%) }\end{array}$ & 0,464 & 7,664 \\
\hline Número de doutores por número de funcionários (\%) & 0,164 & 2,710 \\
\hline $\begin{array}{l}\text { Número de patentes depositadas e/ou concedidas por } \\
\text { número de funcionários }\end{array}$ & 0,140 & 2,309 \\
\hline Percentual de projetos de inovação finalizados (\%) & 0,381 & 6,277 \\
\hline Faturamento por número de funcionários & 0,184 & 3,036 \\
\hline Lucro líquido por número de funcionários & 0,083 & 1,375 \\
\hline Redução de custos por número de funcionários & 0,161 & 2,649 \\
\hline Participação no mercado nacional (\%) & 0,028 & 0,467 \\
\hline
\end{tabular}

Fonte: Andreassi e Sbragia (2001).

A distância máxima entre os dois grupos está associada à variável "número de doutores, mestres e graduados por número de funcionários", que é a primeira variável a entrar no modelo. Mais adiante, no step 1, novas distâncias são calculadas, agora, sem a primeira variável a entrar no modelo e sem as variáveis removidas. As distâncias quadráticas e a estatística $F$ do step 1 se encontram relacionadas na Tabela 8.

\section{Tabela 8}

Distância quadrática e estatística $\mathrm{F}$ para o step 1

\begin{tabular}{lcc}
\hline \multicolumn{1}{c}{ Variável } & $\begin{array}{c}\text { Distância } \\
\text { quadrática }\end{array}$ & Estatística $\boldsymbol{F}$ \\
\hline Despesas em P\&D por faturamento (\%) & 0,544 & 1,148 \\
\hline Número de doutores por número de funcionários (\%) & 0,508 & 0,628 \\
\hline $\begin{array}{l}\text { Número de patentes depositadas e/ou concedidas por } \\
\text { número de funcionários }\end{array}$ & 0,506 & 0,593 \\
\hline Percentual de projetos de inovação finalizados (\%) & 0,831 & 5,308 \\
\hline Faturamento por número de funcionários & 0,646 & 2,618 \\
\hline Lucro líquido por número de funcionários & 0,643 & 2,584 \\
\hline Redução de custos por número de funcionários & 0,562 & 1,407 \\
\hline Participação no mercado nacional (\%) & 0,507 & 0,601 \\
\hline
\end{tabular}

Fonte: Andreassi e Sbragia (2001)

A próxima variável a entrar no modelo é o "percentual de projetos de inovação finalizados". Novas distâncias serão calculadas com as variáveis restantes, conforme consta da Tabela 9.

Tabela 9

Distância quadrática e estatística $\mathrm{F}$ para o step 2

Variável Distância

quadrática

\begin{tabular}{lcc}
\hline Despesas em P\&D por faturamento (\%) & 0,876 & 0,588 \\
\hline Número de doutores por número de funcionários (\%) & 0,894 & 0,823 \\
\hline $\begin{array}{l}\text { Número de patentes depositadas e/ou concedidas por } \\
\text { número de funcionários }\end{array}$ & 0,879 & 0,626 \\
\hline Faturamento por número de funcionários & 1,062 & 3,042 \\
\hline Lucro líquido por número de funcionários & 1,019 & 2,466 \\
\hline Redução de custos por número de funcionários & 0,912 & 1,057 \\
\hline Participação no mercado nacional (\%) & 0,897 & 0,863 \\
\hline
\end{tabular}

Fonte: Andreassi e Sbragia (2001) 
Como as estatísticas $F$ obtidas são menores que o valor default adotado $(3,84)$, o stepwise se encerra no step 2. Assim, foram encontradas duas variáveis independentes ("doutores, mestres e graduados por número de funcionários" e "percentual de projetos finalizados") que discriminam a variável dependente (grau de inovação). Assim, a função discriminante terá os seguintes coeficientes:

Grau de inovação $=0,73623$ (doutores, mestres e graduados $/ \mathrm{n}^{\mathrm{o}}$ func.) $+0,66391$ (projetos finalizados).

Cabe destacar que ao se fazer a matriz de classificação, $63,8 \%$ dos elementos foram corretamente classificados. Para saber se esse percentual é satisfatório ou não, foi aplicado o teste estatístico Press's $Q$, que mostrou que a discriminação das empresas nos dois grupos foi significativamente melhor que a distribuição aleatória. É importante ressaltar que, embora o percentual de elementos corretamente classificados após a aplicação da técnica $(63,8 \%)$ possa parecer baixo, na aplicação da técnica em casos práticos é comum encontrar valores dessa ordem, como por exemplo o estudo de Dant et al. (1990), cujos mesmos percentuais de elementos corretamente classificados variavam de $64 \%$ a $70 \%$, nas várias análises discriminantes efetuadas pelos autores.

Em resumo, os resultados verificados permitem concluir que de todas as variáveis relacionadas no modelo conceitual, somente duas - "número de doutores, mestres e graduados alocados à $\mathrm{P} \& \mathrm{D}$ por número de funcionários" e "percentual de projetos de inovação finalizados" - discriminam as empresas mais inovadoras das menos inovadoras. Tal resultado é confirmado estatisticamente pela aplicação da estatística $Q$. Porém, ao se minimizar o efeito da variável "percentual de projetos de inovação finalizados", pelo fato dessa variável ser de difícil precisão (algumas empresas podem entender como "projeto" uma simples ordem de serviço, enquanto outras podem dar um significado mais amplo ao termo) e pouco encontrada na literatura como indicador de resultado de $\mathrm{P} \& \mathrm{D}$, é possível concluir, por este estudo, que o número de funcionários alocados à $\mathrm{P} \& \mathrm{D}$ é o grande diferencial das empresas mais inovadoras diante das menos inovadoras.

Esse fato pode indicar a importância da empresa manter em seus quadros internos uma equipe técnica voltada para a pesquisa e desenvolvimento. De fato, independentemente da empresa optar entre comprar ou gerar tecnologia, uma equipe técnica especializada e treinada em P\&D é fundamental para se conseguir maior eficácia na inovação in house ou adquirida de terceiros, criando, nesse último caso, ao menos capacidade de "comprar bem". Esses resultados, inclusive, estão de acordo com aqueles encontrados neste artigo, na seção "Comprar ou geral tecnologia?".

\section{Conclusões}

Certamente não se pretendeu esgotar aqui as recomendações a respeito das medidas que as empresas devem tomar para conseguirem melhores resultados em seus esforços pela inovação. O objetivo foi apenas discutir três pontos que o autor acredita serem importantes para uma empresa conseguir um melhor resultado dos seus investimentos em inovação tecnológica: equilíbrio entre comprar e fazer tecnologia, não atrelar investimentos em $\mathrm{P} \& \mathrm{D}$ aos resultados da empresa e manter uma equipe interna dedicada à pesquisa e desenvolvimento.

Entretanto, é importante ressaltar que este estudo foi baseado em outras pesquisas de natureza eminentemente quantitativa, com todas as limitações dessa metodologia. Ao se analisar a inovação através de indicadores, o processo foi visto de uma maneira estática. A realidade mostra que a inovação é um fenômeno complexo e dinâmico, que envolve muitas outras variáveis além daquelas mensuradas pelos indicadores aqui empregados. Nesse sentido, fica a sugestão de que também sejam feitos estudos de natureza qualitativa, para verificar a percepção dos empresários sobre a importância dos três pontos mencionados.

Finalmente, é importante ressaltar que todo cuidado é pouco quanto às conclusões generalizantes, já que é difícil fazer generalizações estatisticamente significativas quando se consideram indicadores de inovação referentes a empresas de diferentes setores e regiões. 


\section{Referências bibliográficas}

ALBUQUERQUE, E. M. Sistemas de inovação, acumulação científica nacional e o aproveitamento de "janelas de oportunidade": notas sobre 0 caso brasileiro. Dissertação (Mestrado) - Faculdade de Administração, Contabilidade e Economia, UFM G. 1995.

ANDREASSI, T. Expenditures in technological innovation: a study in Brazilian companies. Dissertação de Mestrado (Mphil) apresentada no SPRU, Sussex University, 1998. $\bar{F} \bar{E} \bar{A}-\bar{U} S P, 1999$.

Estudo das relações entre indicadores de P\&D e indicadores de resultado empresarial em empresas brasileiras. Tese (doutorado) -

; SBRAGIA, R. Fatores determinantes do grau de inovatividade das empresas: um estudo utilizando a técnica da análise discriminante. In. ENCONTRO DA ASSOCIAÇÃO NACIONAL DOS PROGRAMAS DE PÓS-GRADUAÇÃO EM ADM INISTRAÇÃO (ENANPAD) 25., 2001, Campinas. Anais... Campinas: Anpad, 2001.

ANPEl. Dispêndios empresariais de inovação tecnológica no Brasil: resultados da base de dados Anpei (ano-base 1996). São Paulo, 1997.

BELL, M.; PAVITT, P. Technological accumulation and industrial growth: contrasts between developed and developing countries. Sussex University, 1993. Mimeografado.

The development of technological capabilities. In: HAQUE, I. (Ed.). Trade, technology and international competitiveness. Washington: The World Bank, 1995.

BRENNER, M. S.; RUSHTON, B. M. A strategic analysis of sales growth and R\&D in the chemical industry. Research Technological Management, v.32, n.2, p.8-15, Mar./Apr. 1989.

COHEN, W. M.; LEVINTHAL, D. A. Innovation and learning: the two faces of R\&D. The Economic Journal, v.99, p.569-596, 1989.

COOPER, R. G.; KLEINSCHMIDT, E. J. Winning businesses in product development: the critical success factors. Research Technology Management, Jul./Aug. 1996.

DUGAL, S. S.; M ORBEY, G. K. Revisiting corporate R\&D spending during recession. Research Technology Management, p.23-27, Jul./Aug. 1995.

FRANKO, L.G. Global corporate competition: who's winning, who's losing and the R\&D factor as one reason why. Strategic Management Journal, v.10, p.449-474, 1989.

FREEMAN, C.; SOETE, L. The economics of industrial innovation. London: Pinter Publishers, 1997.

MORBEY, G. K.; REITHNER, R. M. How R\&D affects sales growth, productivity and profitability. Research Technology Management, p.1114, May/Jun. 1990.

ODAGIRI, H. R\&D expenditures, royalty payments and sales growth in japonese manufacturing corporations. Journal of Industrial Economics, v.32, n.1, p.61-71, 1983.

PATEL, P.; PAVITT, K. Patterns of technological activity: their measurement and interpretation. In: STONEMAN, P. (Ed.). Handbook of economics of innovation and technological change, Oxford, 1995. p.14-51. 